\title{
A correlação entre os transtornos alimentares e de autoimagem na adolescência
}

\author{
The correlation between eating and self-image disorders in adolescence \\ La correlación entre los trastornos de la alimentación y la autoimagen en la adolescência
}

Recebido: 14/10/2021 | Revisado: 21/10/2021 | Aceito: 22/10/2021 | Publicado: 24/10/2021

\author{
Elaine Costa da Silva \\ ORCID: https://orcid.org/0000-0002-3616-9372 \\ Centro Universitário Fametro, Brasil \\ E-mail: elacostta18@gmail.com \\ Jasmim da Silva Simões \\ ORCID: https://orcid.org/0000-0001-8934-793X \\ Centro Universitário Fametro, Brasil \\ E-mail: jasmim.silva.simoes@gmail.com \\ José Carlos de Sales Ferreira \\ ORCID: https://orcid.org/0000-0002-1867-8229 \\ Centro Universitário Fametro, Brasil \\ E-mail: jcarlos.sales@gmail.com \\ Rebeca Sakamoto Figueiredo \\ ORCID: https://orcid.org/0000-0002-9819-8099 \\ Centro Universitário Fametro, Brasil \\ E-mail: rebeca.figueiredo@fametro.edu.br
}

\begin{abstract}
Resumo
Introdução: Os transtornos alimentares, por muitas vezes se tornam ciclos viciosos, e podem impactar diretamente na vida biopsicossocial do indivíduo, tendo em vista que este por vezes se isola do convívio com outras pessoas preocupado com a alimentação e a imagem corporal. Objetivo: analisar a relação entre nutrição e transtornos de auto imagem em adolescentes. Metodologia: Para a realização desse estudo optou-se por uma pesquisa na modalidade de revisão integrativa de literatura. A coleta das informações para a pesquisa bibliográfica será por meio da exploração da base de dados da Biblioteca Virtual em Saúde (BVS), Biblioteca Cientifica Eletrônica Virtual (SCIELO) e Literatura Latino - Americana e do Caribe em Ciências da Saúde (LILACS). Resultados e Discussão: Sabe-se que o período da adolescência é caracterizado por diversas alterações morfológicas que são reguladas pelo processo maturacional. Entre essas modificações destaca-se o aumento da gordura corporal e da massa magra em meninas e meninos, respectivamente. A não aceitação destas características pubertárias pode repercutir em problemas na construção da imagem corporal do jovem. Conclusão: Com o desenvolvimento desse estudo pode-se conhecer muitos detalhes e questões sobre os transtornos de auto imagem desenvolvidos entre adolescentes, tendo em vista que essa é uma problemática cada vez mais evidente na sociedade atual.
\end{abstract}

Palavras-chave: Autoimagem; Alimentos; Alimentação e nutrição; Adolescentes; Transtornos.

\begin{abstract}
Introduction: Eating disorders, if not treated, become vicious circles that can significantly impact the social life of the individual, since he ends up isolating himself from living with other people because of his concern exacerbated with food and body image. Objective: to analyze the relationship between nutrition and self-image disorders in adolescents. Methodology: For this study, we opted for a research in the modality of integrative literature review. The collection of information for bibliographic research will be through the exploration of the database of the Virtual Health Library (VHL), Virtual Electronic Scientific Library (SCIELO) and Latin American and Caribbean Literature on Health Sciences (LILACS). Results and Discussion: It is known that the period of adolescence is characterized by several morphological alterations that are regulated by the maturational process. Among these changes, there is an increase in body fat and lean mass in girls and boys, respectively. The non-acceptance of these pubertal characteristics may have repercussions on problems in the construction of the body image of the young person. Conclusion: With the development of this study, many details and questions about self-image disorders developed among adolescents can be known, considering that this is an increasingly evident problem in today's society.
\end{abstract}

Keywords: Self image; Foods; Food and nutrition; Teenagers; Disorders.

\section{Resumen}

Introducción: Los trastornos de la alimentación a menudo se convierten en círculos viciosos y pueden afectar directamente la vida biopsicosocial del individuo, dado que en ocasiones se encuentra aislado de convivir con otras personas preocupadas por la alimentación y la imagen corporal. Objetivo: analizar la relación entre la nutrición y los trastornos de la autoimagen en adolescentes. Metodología: Para la realización de este estudio se optó por una 
investigación en la modalidad de revisión integradora de la literatura. La recolección de información para la investigación bibliográfica se realizará a través de la exploración de la base de datos de la Biblioteca Virtual en Salud (BVS), Biblioteca Virtual Científica Electrónica (SCIELO) y Literatura Latinoamericana y del Caribe en Ciencias de la Salud (LILACS). Resultados y Discusión: Se sabe que el período de la adolescencia se caracteriza por varios cambios morfológicos que son regulados por el proceso de maduración. Entre estos cambios, destaca el aumento de grasa corporal y masa magra en niñas y niños, respectivamente. La no aceptación de estas características puberales puede generar problemas en la construcción de la imagen corporal del joven. Conclusión: Con el desarrollo de este estudio, es posible conocer muchos detalles y preguntas sobre los trastornos de la autoimagen que se desarrollan entre los adolescentes, considerando que este problema es cada vez más evidente en la sociedad actual.

Palabras clave: Auto imagen; Alimentos; Comida y nutrición; Adolescentes; Trastornos.

\section{Introdução}

Atualmente, as pessoas buscam cada vez mais um conhecimento mais aprofundado sobre o comportamento alimentar de indivíduos e comunidades, pois isso depende de como e como cada pessoa interage com os alimentos. A alimentação é uma prática permanente e indispensável à sobrevivência humana, definida como um fenômeno complexo, incluindo aspectos psicológicos, fisiológicos e socioculturais (Kessler \& Poll,2018).

Segundo Vaz (2014), os padrões estéticos corporais promovidos hoje são diferentes dos promovidos no passado, considerando que a magreza e a musculatura são mais definidas como sinais de saúde, beleza e força do que como reflexo da aptidão física. Desnutrição, pobreza e até doenças. De acordo com o relato do autor, esse modelo pode causar depressão e baixa autoestima em pessoas que não atendem a essa regra, levando a transtornos alimentares e outras condições.

Nesse contexto, Alvarenga e Philippi (2011) apontam que nos transtornos alimentares, alimentação irregular, alimentação obsessiva e obsessiva, comportamentos alimentares restritivos e laxativos são muito comuns, sendo considerados síndromes comportamentais de origem multifatorial. Entre eles, os transtornos alimentares mais comuns são a anorexia e a bulimia.

Desta forma, nota-se que os transtornos alimentares quando não tratados, podem se tornar ciclos viciosos que impactam diretamente na vida social do indivíduo, tendo em vista que este se isola do convívio social por conta do ato de ter que alimentar-se e da auto imagem (Palma, Santos \& Ribeiro, 2013). Nesse contexto, é referido por Bandeira et al., (2016), que a imagem corporal é conceituada pela visão que cada indivíduo possui do próprio corpo.

Segundo Fortes et al., (2015), esse conceito é definido de acordo com a particularidade de cada indivíduo, e que o proporciona sensações, pensamentos e percepções distintas acerca do próprio corpo. É óbvio também que a mídia e a moda atuais podem afetar a percepção física e o comportamento alimentar dos jovens, pois o impacto da exposição na mídia sobre eles pode estar relacionado ao aumento dos indicadores de transtorno alimentar e mais atenção aos jovens. Perda de peso (Fortes et al., 2015).

Alves et al., (2012), relaram ao contar com as crenças valorizadas por esses meios de comunicação, os jovens serão levados a acreditar que seu comportamento os ajudará sempre a se adaptarem à realidade, afastando-os de quaisquer sentimentos ou pensamentos que possam ameaçar esse equilíbrio, pois as crenças são entendidas como crenças, e o dogma é considerado verdadeiro e inquestionável.

Os transtornos alimentares mais famosos são a anorexia nervosa e a bulimia nervosa. Ambos são caracterizados por atenção excessiva ao peso, especialmente mulheres jovens. Considerando a complexidade multifatorial associada ao surgimento dessas doenças, as pessoas costumam ter visões negativas sobre a imagem corporal, medo da obesidade e fácil adesão a diferentes métodos inadequados de controle de peso (Silva et al., 2012).

O desenvolvimento desse estudo justifica-se devido ao crescente índice no que se refere ao desenvolvimento de transtorno de auto imagem entre adolescentes. Sabe-se que o padrão de beleza empregado atualmente é tido como surreal, fazendo com que os adolescentes tentem alcançar esses padrões, desenvolvimento assim, transtornos que podem perdurar 
durante toda vida. Com isso, torna-se importante conhecer os aspectos que cercam os hábitos nutricionais bem como a sua correlação com os transtornos de auto imagem desenvolvidos por adolescentes com o objetivo de analisar a relação entre nutrição e transtornos de auto imagem em adolescentes.

\section{Metodologia}

Para a realização desse estudo optou-se por uma pesquisa na modalidade de revisão integrativa de literatura. A revisão integrativa determina o conhecimento atual sobre uma temática específica, já que é conduzida de modo a identificar, analisar e sintetizar resultados de estudos independentes sobre o mesmo assunto (Silveira et al., 2008).

A Revisão Integrativa é um método de pesquisa apontado como ferramenta de grande relevância no campo da saúde, por proporcionar a busca, a avaliação crítica e a síntese de evidências sobre um tema investigado. Esses aspectos facilitam a identificação dos resultados relevantes, de lacunas que direcionam para o desenvolvimento de futuras pesquisas e auxiliam o profissional a escolher condutas e a tomar decisões, proporcionando um saber crítico (Souza, Silva \& Carvalho, 2010).

Para extrair os dados dos artigos selecionados, será necessária a utilização de um instrumento previamente elaborado capaz de assegurar que a totalidade dos dados relevantes seja extraída, minimizar o risco de erros na transcrição, garantir precisão na checagem das informações e servir como registro (Souza, Silva \& Carvalho, 2010).

A coleta das informações para a pesquisa bibliográfica será por meio da exploração da base de dados da Biblioteca Virtual em Saúde (BVS), Biblioteca Cientifica Eletrônica Virtual (SCIELO) e Literatura Latino - Americana e do Caribe em Ciências da Saúde (LILACS). A busca na base de dados será orientada pelos descritores: auto imagem; alimentos, alimentação e nutrição; adolescentes; transtornos; e será realizado em todos os índices, buscando captar o maior número de artigos publicados no período proposto que abordem a temática em discussão.

Para uma análise crítica e reflexiva dos estudos incluídos na revisão, será realizada uma leitura minuciosa e criteriosa destacando os que atingirem os critérios de inclusão e que contemplarem o objetivo proposto, para viabilizar o resultado da pesquisa de forma clara e objetiva. Serão incluídos os artigos publicados em língua portuguesa nos últimos dez anos, com texto completo, disponível on-line, com acesso livre. Serão excluídos da amostra os artigos publicados em línguas estrangeiras, os que não apresentarem o texto na integra, artigos que não apresentavam relação direta com o tema, resumos, monografias, dissertações, teses e artigos repetidos.

\section{Resultados e Discussão}

\subsection{Os principais transtornos de auto imagem presentes entre os adolescentes}

Sabe-se que o período da adolescência é caracterizado por diversas alterações morfológicas que são reguladas pelo processo maturacional. Entre essas modificações destaca-se o aumento da gordura corporal e da massa magra em meninas e meninos, respectivamente. A não aceitação destas características pubertárias pode repercutir em problemas na construção da imagem corporal do jovem (Anderson, 2018).

É citado por Baile et al., (2013) que a insatisfação corporal é um dos critérios diagnósticos dos transtornos alimentares (TAs), sendo os transtornos alimentares doenças psiquiátricas que afetam, na sua maioria, adolescentes e adultos jovens do sexo feminino, podendo levar a grandes prejuízos biológicos e psicológicos e aumento de morbidade e mortalidade.

Becker et al., (2018) ressalta duas entidades nosológicas principais: a Anorexia Nervosa e a Bulimia Nervosa. Embora classificados separadamente, os dois transtornos acham-se intimamente relacionados por apresentarem psicopatologia comum: uma ideia prevalente envolvendo a preocupação excessiva com o peso e a forma corporal (medo de engordar), que leva as pacientes a se engajarem em dietas extremamente restritivas ou a utilizarem métodos inapropriados para alcançarem o corpo 
idealizado. Tais pacientes costumam julgar a si mesmas baseando-se quase que exclusivamente em sua aparência física, com a qual se mostram sempre insatisfeitas.

A anorexia nervosa é um transtorno do comportamento alimentar caracterizado por limitações dietéticas autoimpostas, padrões bizarros de alimentação com acentuada perda de peso induzida e mantida pelo paciente, associada a um temor intenso de tornar-se obeso (Branco et al., 2016).

O termo anorexia deriva do grego - "an", deficiência ou ausência de, e "orexis", apetite, significando, portanto, inapetência ou perda do apetite. Contudo, seu significado etimológico é questionado, visto que, não apresentam real perda de apetite até estágios mais avançados da doença, mas sim uma recusa alimentar deliberada, com intuito de emagrecer ou por medo de engordar (Branco et al., 2016).

Já a bulimia nervosa, é síndrome caracterizada por repetidos ataques de hiperfagia, preocupação excessiva com o controle de peso corporal, levando o paciente a adotar medidas extremas a fim de mitigar os efeitos de engordar da ingestão de alimentos (Campana et al., 2019).

A bulimia é caracterizada por períodos de grande ingestão alimentar de uma maneira muito rápida e com a sensação de perda de controle (episódios bulímicos), durante os quais são consumidos uma grande quantidade de alimentos, em um curto período de tempo. O sentimento de descontrole sobre o comportamento alimentar e preocupação excessiva com o controle do peso corporal faz com que o paciente adote medidas compensativas, afim de evitar o ganho de peso (Campana $e t$ al., 2019). Com isto, nota-se que este comportamento lhe traz satisfação e alívio momentâneos após a hiperalimentação, pois o mesmo acredita ter descoberto a forma ideal de manter o peso sem restringir os alimentos que consideram proibidos.

O tratamento eficiente do TA é a busca de ajuda de uma equipe multidisciplinar composta por médicos, nutricionista, psiquiatra, psicólogo, educador físico, sendo que nele inclui a estabilização nutricional para os padrões saudáveis de peso, a conscientização de quanto esse comportamento é inadequado e prejudicial e até o uso de medicação para controlar os impulsos e quadros de depressão, quando necessário (Branco et al., 2016).

\subsection{Correlação entre os hábitos nutricionais com os transtornos de auto imagem entre adolescentes}

Nos últimos 20 anos, a incidência global de transtornos do comportamento alimentar quase dobrou, o que mostra que as pessoas estão prestando cada vez mais atenção à sua imagem corporal. É preocupante o número de adolescentes que expressam insatisfação com sua imagem corporal, e esse problema não atinge apenas um determinado gênero ou faixa etária, mas sim em adolescentes de todas as faixas etárias, tanto homens quanto mulheres (Sousa et al., 2016).

Segundo Conti et al., (2014), em relação ao gênero, o desejo das mulheres obviamente é perder peso, enquanto o desejo dos homens é justamente o contrário, ganhar peso. Em outras palavras, as meninas tendem a evitar a gordura corporal, enquanto os meninos querem mais massa muscular. Portanto, devemos lembrar que a insatisfação com o corpo na adolescência não é homogênea.

O incentivo a hábitos de vida saudáveis, como uma boa alimentação combinada com exercícios físicos regulares, pode prevenir a insatisfação e muitas outras doenças a ela relacionadas. Isso é muito óbvio em alguns dos estudos aqui analisados. De qualquer forma, é admirável que se realizem medidas que visem a prevenção, bem como o esclarecimento acerca da importância dos hábitos saudáveis e sobre os aspectos culturais que cercam o corpo humano, bem como a forma como ele é enxergado e suas formas de tratamento (Monteiro et al. 2014).

A insatisfação é, na verdade, causada por múltiplos fatores, pois se refere à forma como os indivíduos interagem com o meio em que vivem e como se percebem nesse meio. Portanto, o fato de estar insatisfeito com a cultura, família e amigos gera reflexos na satisfação corpórea (Santiago et al., 2015). 
Outro fator que vincula a insatisfação física com o curso das doenças de saúde é que, em muitos casos, a insatisfação decorre de problemas de saúde como obesidade ou sobrepeso, que por sua vez podem ser uma alimentação inadequada e estilo de vida sedentário e outros produtos. coisa. Portanto, vemos que a insatisfação com a imagem corporal é muito importante, antes mesmo de aparecer (Pereira, Souza \& Monteiro, 2017).

Entre muitos outros fatores, este está diretamente relacionado à forma como o indivíduo vê seu corpo e como o avalia, podendo-se citar a baixa autoestima, a idade, os fatores relacionados ao peso, cor, etc. Pode-se citar ainda, fatores externos que contribuem igualmente para a insatisfação corporal, como a preocupação de ser excluído do grupo, rejeitado e desvalorizados e até mesmo discriminado (Fidelix et al., 2018).

\subsection{As consequências do desenvolvimento dos transtornos de auto imagem entre os adolescentes}

Como todos sabemos, a exploração atual do corpo ideal é seguir os requisitos obrigatórios existentes criados e mantidos pela sociedade. O padrão de beleza admirado pela sociedade vem sendo divulgado e divulgado pela mídia, o que causa grande repercussão no público jovem. A resposta a isso pode ser a superlotação da academia e mudanças nos hábitos alimentares, sejam eles inofensivos (aumento da dieta e ingestão de alimentos leves) ou prejudiciais (jejum prolongado, uso de laxantes, diuréticos, bulimia e anorexia) (Pereira et al., 2016).

Esse problema tem impacto direto no processo saúde-doença dos adolescentes. Porque, como todos sabemos, os adolescentes insatisfeitos com sua imagem costumam adotar métodos não saudáveis ou não saudáveis para controlar o peso e aumentar a massa muscular. Além de causar sérios danos à saúde, essas práticas também podem levar ao surgimento de outras doenças, como a anorexia ou a bulimia nervosa (Silva et al., 2012).

Monteiro (2016) mencionou que outro fator que vincula a insatisfação com a saúde do corpo é que, em muitos casos, a insatisfação decorre de problemas de saúde como obesidade ou sobrepeso, que por sua vez podem ser produto de reações adversas. Hábitos alimentares, estilo de vida sedentário, etc. Portanto, vemos que a insatisfação com a imagem corporal é muito importante, antes mesmo de aparecer.

Segundo Priano (2015), a insatisfação se expressa de forma diferente em mulheres e homens. Entre as mulheres, a perda de peso (gordura) é preferida, enquanto os homens buscam ganhar peso (massa muscular). Essa diferença também existe na forma como eles percebem seu corpo: as mulheres tendem a superestimar seu peso, enquanto os homens tendem a subestimar seu peso.

Isso mostra que a insatisfação com a imagem corporal, em suma, é a insatisfação com o corpo real, se comparado ao padrão ideal de comunicação midiática. Trata-se de um grave problema de saúde pública, pois sua existência pode levar os indivíduos ao risco de comportamentos saudáveis, que estão associados a discursos que associam saúde ao "tipo corporal", e serão excluídos dos grupos desvalorizados. O medo é utilizado como “combustível” entre outros (Sabá et al., 2014).

\section{Considerações Finais}

Com o desenvolvimento desse estudo pode-se conhecer muitos detalhes e questões sobre os transtornos de auto imagem desenvolvidos entre adolescentes, tendo em vista que essa é uma problemática cada vez mais evidente na sociedade atual, sendo tido ainda como fatores tão presentes pela influência midiática, através de padrões corporais pré-estabelecidos, diversas dietas que não contribuem para a qualidade de vida e sim para a disfunção orgânica corporal.

É importante frisar que os hábitos alimentares, bem como a relação que o adolescente tem com a comida, exercem um papel importantíssimo no desenvolvimento desses transtornos, tendo em vista toda a influência que a mídia exerce sobre a evolução dos mesmos, e até a influência das redes sociais na vida dos adolescentes. 


\section{Referências}

Almeida, V. L. J., Araujo, M. B., Jesus, G. A., et al. (2017). Distúrbios alimentares: bulimia e anorexia. Revista Scientia Plena Jovem, 5(1), $32-36$.

Alvarenga, M., \& Philippi, S. T. (2018). Nutrição e transtornos alimentares: avaliação e tratamento. Manole.

Alvarenga, M. S., Scagliusi, F. B., \& Philippi, S. T. (2017). Comportamento de risco para transtorno alimentar em universitárias brasileiras. Rev Psiq Clin., 38(1), 3-7.

Alves, T. C. H. S., Santana, M. L. P., Silva, R. C. R., et al. (2015). Fatores associados a sintomas de transtornos alimentares entre escolares da rede pública da cidade do Salvador, Bahia. J Bras Psiquiatr. 61(2), 55-63.

Bandeira, Y. E. R.; Mendes, A. L. R. F., Cavalcante, A.C.M., \& Arruda, S. P. M. (2016). Avaliação da imagem corporal de estudantes do curso de Nutrição de um centro universitário particular de Fortaleza. J Bras Psiquiatr. 65(2), 168-73.

Becker A. E., Burwell R. A., Herzog D. B., Hamburg P., \& Gilman S. E. (2016). Eating behaviors and attitudes following prolonged exposure to television among ethnic Fiji adolescents girls. Br J Psychiatry, 180, 509-514.

Bittencourt, L. J., \& Almeida, R. A. (2018). Transtornos Alimentares: patologia ou estilo de vida. Psicologia \& Sociedade, 25(1), $220-229$.

Bosi, M. L. M., Nogueira, J. A. D., Uchimura, K.Y., et al. (2016). Comportamento alimentar e imagem corporal entre estudantes de medicina. Rev Bras Educ Med., 38(2), 243-52.

Bosi, M. L. M., Luis, R. R., Morgado, C. M. C., et al. (2016). Autopercepção da imagem corporal entre estudantes de nutrição: um estudo no município do Rio de Janeiro. J Bras Psiquiatr, 55(2), 108-113.

Bighetti F., Santos C. B., Santos J. E., \& Ribeiro R. P. P. (2016). Tradução e validação do Eating Attitudes Test em adolescentes do sexo feminino de Ribeirão Preto-SP. J Bras Psiquiatr, 53(6), 339-346

Coqueiro R. S., Petroski E. L., Pelegrini A., \& Barbosa A. R. (2018). Insatisfação com a imagem corporal: avaliação comparativa da associação com estado nutricional em universitários. Rev. Psiquiatr RS, 30(1), 31-38.

Cooper P. J., Taylor M. J., Cooper Z., \& Fairbum C. G. (2017). The development and validation of the body sahoe questionnaire. Int J Eat Disord, 6(4), 485594.

Cordás T. A., \& Neves J. E. P. (2019). Escalas de avaliação de transtornos alimentares. Rev Psiq Clin, 26(N. Esp. 1), $41-48$.

Carvalho, P. H. B.; Filgueiras, J. F.; Neves, C. M., et al. (2017). Checagem corporal, atitude alimentar inadequada e insatisfação com a imagem corporal de jovens universitários. J Bras Psiquiatr. 62(2), 108-14.

Choi, P. Y. L., Pope, H. G., \& Olivardia, R. (2018). Muscle dysmorphia: a new syndrome in weightlifters. British Journal of Sports Medicine. Victoria, 36, 375-376.

Claumann, G. S., Pereira, E. F., Inácio, S., et al. (2017). Body image satisfaction in freshmen college students in physical education courses. Rev Educ Fis, UEM, 25(4), 575-83.

Coqueiro, R. S., Petroski, E. L., Pelegrini, A., \& Barbosa, A. R. (2018). Insatisfação com a imagem corporal: avaliação comparativa da associação com estado nutricional em universitários. Rev. Psiquiatr RS, 30(1), 31-38.

Coutinho, V. F., Azevedo, C. H., Nunes, M. L., et al. (2017). Relação do estado nutricional com a percepção e a satisfação da imagem corporal de adolescentes inseridos em programa de inclusão social em Porto Alegre, RS. Revista EFDeportes. Buenos Aires, $15,166$.

Duarte, F. M., Almeida, S. D. S., \& Martins, K. A. (2018). Alimentação fora do domicílio de universitários de alguns cursos da área da saúde de uma instituição privada. Mundo Saúde, 37(3), 288-98.

Fortes, L. S., Ferreira, M. E. C., Costa, P. R. F, et al. (2017). Comparação do comportamento de risco para transtornos alimentares entre adolescentes atletas e não atletas. J Bras Psiquiatr., 4(4), 296-302.

Fortes, L. S., Filgueiras, J. F., \& Ferreira, M. E. C. (2016). Comportamentos de risco para transtornos alimentares e sintomas depressivos: um estudo com jovens do sexo feminino de Juiz de Fora, Minas Gerais, Brasil. Cad Saúde Pública, 30(11), 2443-50.

Fortes, L. S., Almeida, S. S., \& Ferreira, M. E. C. (2018). Impacto de variáveis antropométricas sobre a insatisfação corporal e o comportamento alimentar em jovens atletas. J Bras Psiquiatr., 61(4), 235-41.

Frank, R., Claumann, G. S., Pinto, A. A., et al. (2016). Fatores associados à insatisfação com a imagem corporal em acadêmicos de Educação Física. J Bras Psiquiatr, 65(2), 161-7.

Gonçalves, V. O., \& Martínez, J. P. (2017). Imagem corporal de adolescentes: um estudo sobre as relações de gênero e influência da mídia. Comunicação e Informação, 17(2), 139-154.

Guedes, D. P. (2018). Composição corporal: princípios, técnicas e aplicações. Santa Catarina: CEITEC.

Heo, J., Oh, J., Subramanian, S.V., Kim, Y. (2018). Addictive internet use among Korean adolescents: a national survey. PLOS One, 9(2), 1-8.

Kravchychyn, A. C. P., Silva, D. F., \& Machado, F. A. (2019). Relação entre estado nutricional, adiposidade corporal, percepção de autoimagem corporal e risco para transtornos alimentares em atletas de modalidades coletivas do gênero feminino. Rev Bras Educ Fís Esporte, 27(3), 459-66. 
Kretschmer, A. C., Rodrigues, G. O., Ristow, A. S., \& Peixoto, N. C. (2016). Estado nutricional e hábitos alimentares de acadêmicos de uma universidade do norte do Rio Grande do Sul. Rev Saúde (Santa Maria), 41(2), 121-8.

Kotait, M. S., Barillari, M. L., \& Conti, M. A. (2020). Escalas de avaliação de comportamento alimentar. In: Cordás TA, Kachani AT. Nutrição em psiquiatria. Porto Alegre: Artmed.

Kakeshita I. S., \& Almeida S. S. (2016). Relação entre índice de massa corporal e a percepção da auto-imagem em universitários. Rev Saude Publica, 40(2), 497-504.

Lohman, T. G., Roche, A. F., Martorell, R., editors. (2018). Anthropometric standardization reference manual. Champaign: Human Kinetics Books.

Liao Y., Knoesen, N. P., Castle, D. J., Tang, J., Deng, Y., Bookun, R., Chen, X., Hao, W., Meng, G., \& Liu, T. (2020). Symptoms of disordered eating, body shape, and mood concerns in male and female Chinese medical students. Compr Psychiatry, 51(1), 516-523.

Lazareth, R. A., Silva, T. R., Palombo, V., \& Navarro, F. (2019). Perfil antropométrico, metabólico e hemodinâmico de atletas adolescentes de 14 a 19 anos de idade. Revista Brasileira de Nutrição Esportiva. 4(20). 173-181.

Leal, G. V. S., Philippi, S. T., Polacow, V. O., et al. (2017). O que é comportamento de risco para transtornos alimentares em adolescentes? J Bras Psiquiatr, $62(1), 62-75$.

Lira, A. G., Ganen, A. P., Lodi, A. S., \& Alvarenga, M. S. (2017). Uso de redes sociais, influência da mídia e insatisfação com a imagem corporal de adolescentes brasileiras. J Bras Psiquiatr, 66(3), 164-71.

Marconato, M. S. F., Silva, G. M. M., \& Frasson, T. Z. (2016). Hábito alimentar de universitários iniciantes e concluintes do curso de nutrição de uma universidade do interior paulista. Rev Bras Obes Nutr Emagrecimento, 10(58), 180-8.

Martins, B. G., Silva, W. R., \& Campos, J. A. D. B. (2015). Preocupação com a forma do corpo de graduandos de Farmácia-Bioquímica. J Bras Psiquiatr. 64(1), 32-9.

Martins, C. R., \& Petroski, E. L. (2015). Insatisfação com a imagem corporal em adolescentes do sexo feminino de uma cidade de pequeno porte: prevalência e correlações. Motri.11(2), 94-106.

Medeiros, T. H., Caputo, E. L., \& Domingues, M. R. (2017). Insatisfação corporal em frequentadoras de academia. J Bras Psiquiatr.66(1):38-44.

Nunes, A. M., Olinto, M. T. A., Barros, F. C., \& Camey, S. (2017). Influência da percepção do peso e do índice de massa corporal nos comportamentos alimentares anormais. Rev Bras Psiquiatr, 23(1), 21-27.

Organização Mundial Da Saúde (OMS). (2018). Obesity -Presenting and managing the global epidemic. Report of a WHO consultation on obesiy. Genebra: OMS.

Palma, R. F. M., Santos, J. E., \& Ribeiro, R. P. P. (2018). Evolução nutricional de pacientes com transtornos alimentares: experiência de 30 anos de um Hospital Universitário. Rev Nutr, 26(6), 669-78.

Penz, L. R., Dal Bosco, S. M., \& Vieira, J. M. (2018). Risco para desenvolvimento de transtornos alimentares em estudantes de Nutrição. Scientia Medica; $18(3), 124-128$.

Reis, J. A., Silva, J. C. R. R., \& Pinho, L. (2016). Fatores associados ao risco de transtornos alimentares entre acadêmicos da área de saúde. Rev Gaúcha Enferm., 35(2), 73-8.

Santos, C. A., Motta, G. S., \& Oliveira, T. C. M. (2018). Transtorno do comportamento alimentar em atletas: distorção da imagem corporal, incidência, consequências e tratamentos para anorexia e bulimia nervosa. Revista Brasileira de Nutrição Esportiva. São Paulo, 2 (10), 19.

Silva, T. A. B., Ximenes, R. C. C., \& Holanda, M. A. (2016). Frequência de comportamentos alimentares inadequados e sua relação com a insatisfação corporal em adolescentes. J Bras Psiquiatr, 61(3), 154-8.

Silva, T. A. B., Vasconcelos, F. M. N., \& Ximenes, R. C. C. (2019). As terapias cognitivo-comportamentais no tratamento da bulimia nervosa: uma revisão. J Bras Psiquiatr, 64(2), 160-8.

Silva, J. D., Silva, A.B.J., \& Oliveira, A. V. K. (2017). Influência do estado nutricional no risco para transtornos alimentares em estudantes de nutrição. Ciênc Saúde Coletiva. 17(12), 3399-4006.

Souza, A. C., \& Alvarenga, M. S. (2016). Insatisfação com a imagem corporal em estudantes universitários - Uma revisão integrativa. J Bras Psiquiatr, 65(3), 286-99.

Uzunian, L. G., Vitalle, M. S. S. (2015). Habilidades sociais: fator de proteção contra transtornos alimentares em adolescentes. Ciênc Saúde Coletiva, 20(11), 3495-508.

Vaz, D. S. S., \& Bennemann, R. M. (2017). Comportamento alimentar e hábito alimentar: uma revisão. Uningá Rev., 20(1), 108-12. 